\title{
EXISTENCE OF A DEGENERATE SINGULARITY IN THE HIGH ACTIVATION ENERGY LIMIT OF A REACTION-DIFFUSION EQUATION
}

\author{
GEORG S. WEISS AND GUANGHUI ZHANG
}

ABSTRACT. We consider the singular perturbation problem

$$
\Delta u_{\epsilon}=\beta_{\epsilon}\left(u_{\epsilon}\right),
$$

where $\beta_{\epsilon}(s)=\frac{1}{\epsilon} \beta\left(\frac{s}{\epsilon}\right), \beta$ is a Lipschitz continuous function such that $\beta>0$ in $(0,1), \beta \equiv 0$ outside $(0,1)$ and $\int_{0}^{1} \beta(s) d s=\frac{1}{2}$.

We construct an example exhibiting a degenerate singularity as $\epsilon_{k} \searrow 0$. More precisely, there is a sequence of solutions $u_{\epsilon_{k}} \rightarrow u$ as $k \rightarrow \infty$, and there exists $x^{0} \in \partial\{u>0\}$ such that

$$
\frac{u\left(x^{0}+r \cdot\right)}{r} \rightarrow 0 \text { as } r \rightarrow 0 .
$$

Known results suggest that this singularity must be unstable, which makes it hard to capture analytically and numerically. Our result answers a question raised by Jean-Michel Roquejoffre at the FBP'08 in Stockholm.

\section{INTRODUCTION}

In this paper we are concerned with the singular perturbation problem

$$
\Delta u_{\epsilon}=\beta_{\epsilon}\left(u_{\epsilon}\right) \text { in } \Omega
$$

where $\Omega$ is a bounded domain in $\mathbb{R}^{n}, \epsilon>0$ and $\beta_{\epsilon}(s)=\frac{1}{\epsilon} \beta\left(\frac{s}{\epsilon}\right)$. Here $\beta$ is a Lipschitz continuous function such that $\beta>0$ in $(0,1), \beta \equiv 0$ outside $(0,1)$ and $\int_{0}^{1} \beta(s) d s=\frac{1}{2}$.

2000 Mathematics Subject Classification. Primary 35R35, Secondary 35J60.

Key words and phrases. Free boundary, singular point, unstable singularity.

G.S. Weiss has been partially supported by the Grant-in-Aid 18740086 of the Japanese Ministry of Education, Culture, Sports, Science and Technology. 
This problem arises in the mathematical analysis of equidiffusional flames (see [3], 4] ), in which case $\epsilon$ is proportional to the inverse of the activation energy.

Formally, as $\epsilon \rightarrow 0$, the solutions $u_{\epsilon}$ converge to a solution $u$ of the free boundary problem

$$
\begin{array}{ll}
\Delta u=0 & \text { in } \Omega \backslash \partial\{u>0\}, \\
u=0,\left(u_{\nu}^{+}\right)^{2}-\left(u_{\nu}^{-}\right)^{2}=1 & \text { on } \partial\{u>0\} .
\end{array}
$$

On a rigorous level, L. Caffarelli established a locally uniformly Lipschitz estimate for bounded solutions $\left\{u_{\epsilon}\right\}$ (see [5]). In [10], C. Lederman and N. Wolanski proved that $u$ is a viscosity solution of (1.2). They also proved that $u$ satisfies the free boundary condition in a pointwise sense at non-degenerate free boundary points at which there is an inward unit normal of $\{u>0\}$ in the measure theoretic sense. Here "non-degenerate" means that

$$
r^{-n-1} \int_{B_{r}(x)} u^{+} \geq c>0 \text { for } r \leq r_{0} .
$$

A related convergence result has been proved for the $p$-Laplace operator in [6].

From [1, Lemma 3.4] we know that all free boundary points of local minimizers are non-degenerate. This suggests that if there are degenerate free boundary points of a limit function $u$, then they must be unstable.

It has been known that cross-shaped free boundaries occur for domain variation solutions (see the Introduction of [12]), for example

$$
u\left(x_{1}, x_{2}\right)=\left|x_{1}^{2}-x_{2}^{2}\right| \text { (cf. Figure 1) }
$$

John Andersson suggested a triple junction example (see Figure 2) where the solution is homogeneous of degree $3 / 2$; domain variation solutions with a degree of homogeneity $\in(1,3 / 2)$ are not possible.

However it is not so obvious whether these examples arise as limits of the reaction-diffusion equation (1.1). This is the question Jean-Michel Roquejoffre posed at the FBP'08 in Stockholm. The main result of the present paper gives a partial answer to that question: 


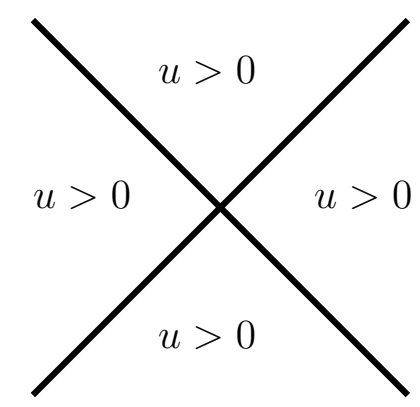

Figure 1. Cross-like Singularity

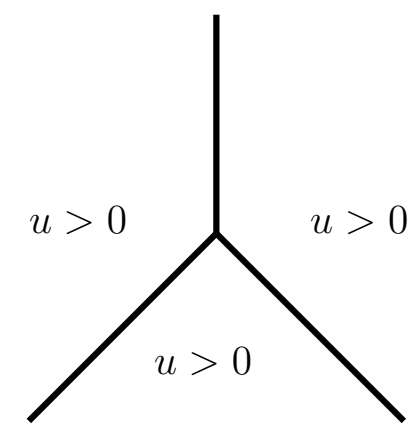

FiguRE 2. Triple Junction Singularity

Theorem A. There exist uniformly bounded solutions $u_{\epsilon_{k}}: B_{1}(0) \subset$ $\mathbb{R}^{2} \rightarrow \mathbb{R}$ such that $\epsilon_{k} \rightarrow 0, u_{\epsilon_{k}} \rightarrow u$ as $k \rightarrow \infty$, and there is a degenerate free boundary point of $u$ in $B_{1}(0)$. More precisely, there is $x^{0} \in B_{1}(0) \cap$ $\partial\{u>0\}$ such that

$$
\lim _{r \rightarrow 0} \frac{u\left(x^{0}+r x\right)}{r}=0 \text { for every } x \in \mathbb{R}^{2} .
$$

Remark 6.5 shows that we may construct degenerate points with an arbitrarily high number of symmetry lines. See Figure 3 for an example with 4 symmetry lines.

Section 3 also suggests a numerical approach for the construction of this unstable solution which would be otherwise hard to capture.

\section{NotAtion}

Throughout this article $\mathbb{R}^{n}$ will be equipped with the Euclidean inner product $x \cdot y$ and $B_{r}\left(x^{0}\right)$ will denote the open ball of center $x^{0}$ with 


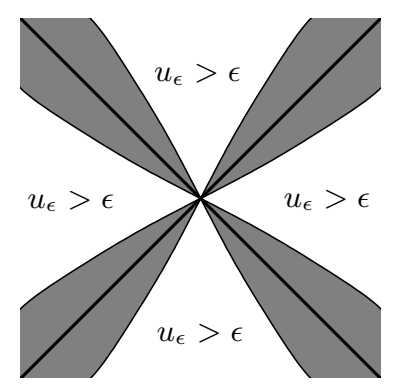

Figure 3. Imaginable $\epsilon$-solution

radius $r$. When not specified, $x^{0}$ is assumed to be 0 . We will use the polar coordinates $(r, \theta)$ in $\mathbb{R}^{2}$. We use the $n$-dimensional Lebesgue measure $\mathcal{L}^{n}$ and the $m$-dimensional Hausdorff measure $\mathcal{H}^{m}$. We shall often use abbreviations for inverse images like $\{u>0\}:=\{x \in \Omega$ : $u(x)>0\}$.

\section{Construction of $\epsilon$-SOlutions}

We are going to use a method introduced in [2].

Let $K=\{(r \cos \theta, r \sin \theta): 0<r<1,0<\theta<\pi / m\}$, where $m$ is an integer $\geq 3$. For $g \in C^{\alpha_{0}}\left(\partial B_{1} \cap \partial K\right), \alpha_{0} \geq \alpha>0, C_{g}^{\alpha}(\bar{K})$ will denote the subspace of $C^{\alpha}(\bar{K})$ consisting of all functions with boundary values $g$ on $\partial B_{1} \cap \partial K$. We consider the following problem:

$$
\begin{array}{ll}
\Delta u(x)=\beta_{\epsilon}(u(x)-u(0)+\epsilon) & \text { in } K, \\
u=g & \text { on } \partial K \cap \partial B_{1}, \\
\frac{\partial u}{\partial \nu}=0 & \text { on } \partial K \backslash \partial B_{1} .
\end{array}
$$

Let $T=T_{\epsilon, g}: C_{g}^{\alpha}(\bar{K}) \rightarrow C_{g}^{\alpha}(\bar{K})$ be the operator defined by

$$
\begin{array}{ll}
\Delta T(u)=\beta_{\epsilon}(u(x)-u(0)+\epsilon) & \text { in } K, \\
T(u)=g & \text { on } \partial K \cap \partial B_{1}, \\
\frac{\partial T(u)}{\partial \nu}=0 & \text { on } \partial K \backslash \partial B_{1} .
\end{array}
$$

Using Schauder's fixed point theorem, we can prove the existence of a solution of equation (3.1): 
Proposition 3.1. For each $g \in C^{\alpha_{0}}\left(\partial B_{1} \cap \partial K\right)$, $T$ has a fixed point $u_{\epsilon} \in C_{g}^{\alpha}(\bar{K})$ for some $\alpha>0$ depending only on $\alpha_{0}$.

Proof. Let $v \in C_{g}^{\alpha}(\bar{K}), F(x)=\beta_{\epsilon}(v(x)-v(0)+\epsilon)$. Then there is a $W^{1,2}(K)$-solution $u$ of the problem:

$$
\begin{array}{ll}
\Delta u=F & \text { in } K, \\
u=g & \text { on } \partial K \cap \partial B_{1}, \\
\frac{\partial u}{\partial \nu}=0 & \text { on } \partial K \backslash \partial B_{1} .
\end{array}
$$

We extend $u$ to a function $\tilde{u}: B_{1} \backslash\{0\} \rightarrow \mathbb{R}$ by even reflection:

$$
\begin{array}{ll}
\tilde{u}(r, \pi / m+\theta)=u(r, \pi / m-\theta), & \text { if } 0 \leq \theta \leq \pi / m, \\
\tilde{u}(r, j \pi / m+\theta)=\tilde{u}(r, j \pi / m-\theta), & \text { if } 0 \leq \theta \leq \pi / m,
\end{array}
$$

for $j=1,2, \ldots, 2 m-1$. It follows that $\tilde{u}$ is a solution of

$$
\begin{array}{ll}
\Delta \tilde{u}=\tilde{F} & \text { in } B_{1} \backslash\{0\}, \\
\tilde{u}=\tilde{g} & \text { on } \partial B_{1},
\end{array}
$$

where $\tilde{F}$ and $\tilde{g}$ are defined by even reflection. As the origin is a set of vanishing 2-capacity (see [7]), $\tilde{u}$ is a weak solution of $\Delta \tilde{u}=\tilde{F}$ in $B_{1}$.

Applying the regularity theory of elliptic equations (see for example [8, Corollary 9.29]), we see that $\tilde{u} \in C^{\alpha}\left(\bar{B}_{1}\right)$ for small $\alpha$, and that $\|\tilde{u}\|_{C^{0, \alpha}\left(B_{1}\right)} \leq C$, where $\alpha \in\left(0, \alpha_{0}\right)$ and $C$ are constants depending on $\alpha_{0},\|g\|_{C^{0, \alpha_{0}}}$ and $\|F\|_{L^{\infty}(K)}$. Hence $T$ is a continuous compact linear operator from $C_{g}^{\alpha}(\bar{K})$ into itself for small $\alpha>0$, and

$$
\left\|T_{\epsilon, g}(w)\right\|_{C^{\alpha}(\bar{K})} \leq C
$$

for every $\sigma \in[0,1]$ and every solution of the equation

$$
\begin{array}{ll}
\Delta w=\sigma \beta_{\epsilon}(w(x)-w(0)+\epsilon) & \text { in } K, \\
w=g & \text { on } \partial K \cap \partial B_{1}, \\
\frac{\partial w}{\partial \nu}=0 & \text { on } \partial K \backslash \partial B_{1}
\end{array}
$$

here $C$ is a constant depending only on $g, \beta$ and $\epsilon$.

From Schauder's fixed point theorem (see [8, Chapter 11]) we infer that $T_{\epsilon, g}$ has a fixed point $u_{\epsilon} \in C_{g}^{\alpha}(\bar{K})$. 


\section{Convergence}

From now on all of our functions are defined in $B_{1}(0)$ by the above reflection. We choose one non-constant $g \in C^{2}(\bar{B})$.

Let $v_{\epsilon}(x)=u_{\epsilon}(x)-u_{\epsilon}(0)+\epsilon$. Then $v_{\epsilon}$ is a solution of

$$
\begin{array}{ll}
\Delta v_{\epsilon}(x)=\beta_{\epsilon}\left(v_{\epsilon}(x)\right) & \text { in } B_{1}, \\
v_{\epsilon}(0)=\epsilon, & \\
v_{\epsilon}=g-u_{\epsilon}(0)+\epsilon & \text { on } \partial B_{1} .
\end{array}
$$

By the maximum principle, $\left|u_{\epsilon}(0)\right| \leq\|g\|_{L^{\infty}\left(B_{1}\right)}$, therefore $\left\|v_{\epsilon}\right\|_{L^{\infty}\left(\bar{B}_{1}\right)} \leq$ $C$, where $C$ depends only on $\|g\|_{L^{\infty}\left(B_{1}\right)}$.

First we state a result from [5] proving a uniform Lipschitz estimate.

Proposition 4.1. Let $w_{\epsilon}$ be a family of solutions to $\Delta w_{\epsilon}=\beta_{\epsilon}\left(w_{\epsilon}\right)$ in a domain $\Omega \subset \mathbb{R}^{n}$ such that $\left\|w_{\epsilon}\right\|_{L^{\infty}(\Omega)} \leq C$ for some $C<\infty$. Let $K \subset \Omega$ be a compact set and let $\tau>0$ be such that $B_{\tau}\left(x^{0}\right) \subset \Omega$ for every $x^{0} \in K$. Then there exists a constant $L=L(\tau, C)$, such that

$$
\left|\nabla w_{\epsilon}(x)\right| \leq L \quad \text { for } x \in K
$$

If the boundary data is smooth enough, we have the following gradient estimate near the boundary (see [9]).

Proposition 4.2. Let $\Omega \subset \mathbb{R}^{n}$ be a bounded domain with smooth boundary and let $w_{\epsilon} \in C^{2}(\Omega) \cap C^{0}(\bar{\Omega})$ be a solution of

$$
\begin{array}{ll}
\Delta w_{\epsilon}(x)=\beta_{\epsilon}\left(w_{\epsilon}(x)\right) & \text { in } \Omega, \\
w_{\epsilon}=f & \text { on } \partial \Omega,
\end{array}
$$

where $f \in C^{2}(\bar{\Omega}),\|f\|_{C^{2}(\bar{\Omega})}<C_{1}$. Then there exist $\epsilon_{0}>0, C<\infty$ such that

$$
\left|\nabla w_{\epsilon}(x)\right| \leq C(1+|\log d(x, \partial \Omega)|)
$$

for $\epsilon \in\left(0, \epsilon_{0}\right), x \in \Omega$, where $d(x, \partial \Omega)$ is the distance from $x$ to the boundary of $\Omega$.

Now we are ready to prove the convergence of $\left\{v_{\epsilon_{i}}\right\}$.

Proposition 4.3. There exist a sequence $\left\{v_{\epsilon_{i}}\right\}$ and a locally Lipschitz continuous function $v_{0}$ such that

1) $v_{\epsilon_{i}} \rightarrow v_{0}$ uniformly on $\bar{B}_{1}$, 
2) $v_{\epsilon_{i}} \rightarrow v_{0}$ in $W_{\mathrm{loc}}^{1,2}\left(B_{1}\right)$,

3) $v_{0}$ is harmonic in $B_{1} \backslash \partial\left\{v_{0}>0\right\}$,

4) $\Delta v_{\epsilon_{i}} \rightarrow \mu$ as measures on $B_{1}$; here $\mu$ is a locally finite non-negative measure supported on the free boundary $\partial\left\{v_{0}>0\right\}$. Therefore

$$
\Delta v_{0}=\mu \quad \text { in } B_{1},
$$

that is

$$
\int_{B_{1}} \nabla v_{0} \cdot \nabla \phi d x+\int_{B_{1}} \phi d \mu=0
$$

for every $\phi \in C_{0}^{1}\left(B_{1}\right)$.

5) $v_{0}(r, j \pi / m+\theta)=v_{0}(r, j \pi / m-\theta)$ for $0 \leq \theta \leq \pi / m$ and $1 \leq j \leq 2 m$.

Proof. 1) By Proposition 4.2, $\left\{v_{\epsilon}\right\}$ is equicontinuous. Since $\left\{v_{\epsilon}\right\}$ is uniformly bounded in $C^{0}\left(\bar{B}_{1}\right)$, by the Arzela-Ascoli theorem there is a sequence $\left\{v_{\epsilon_{i}}\right\}$ that converges to some $v_{0}$ in $C^{0}\left(\bar{B}_{1}\right)$. By Proposition 4.1. it is easy to see that $v_{0}$ is locally Lipschitz continuous.

2) For $0 \leq \eta \in C_{0}^{\infty}\left(B_{1}\right)$, we obtain from the local Lipschitz bound that

$$
\int_{B_{1}} \eta\left|\nabla v_{0}\right|^{2} d x \leq \liminf _{\epsilon_{i} \rightarrow 0} \int_{B_{1}} \eta\left|\nabla v_{\epsilon_{i}}\right|^{2} d x .
$$

If we prove the converse inequality, the result will follow. Multiplying the $\epsilon_{i}$-equation (1.1) by $\eta v_{\epsilon_{i}}$, we obtain that

$$
\int_{B_{1}} v_{\epsilon_{i}} \nabla \eta \cdot \nabla v_{\epsilon_{i}} d x+\int_{B_{1}} \eta\left|\nabla v_{\epsilon_{i}}\right|^{2} d x=-\int_{B_{1}} \eta v_{\epsilon_{i}} \beta_{\epsilon_{i}}\left(v_{\epsilon_{i}}\right) d x \leq 0 .
$$

Letting $\epsilon_{i} \rightarrow 0$, we get

$$
\limsup _{\epsilon_{i} \rightarrow 0} \int_{B_{1}} \eta\left|\nabla v_{\epsilon_{i}}\right|^{2} d x \leq-\int_{B_{1}} v_{0} \nabla \eta \cdot \nabla v_{0} d x .
$$

For each $\delta>0$, we define

$$
v_{\delta}(x)= \begin{cases}v_{0}(x)-\delta, & \text { if } v_{0}(x)>\delta, \\ v_{0}(x)+\delta, & \text { if } v_{0}(x)<-\delta, \\ 0 & \text { otherwise }\end{cases}
$$


Multiplying the $\epsilon_{i}$-equation by $\eta v_{\delta}$ and integrating, we have

$$
\int_{B_{1}} v_{\delta} \nabla \eta \cdot \nabla v_{\epsilon_{i}} d x+\int_{B_{1}} \eta \nabla v_{\delta} \cdot \nabla v_{\epsilon_{i}} d x=0 .
$$

Letting $\epsilon_{i} \rightarrow 0$, it follows that

$$
\int_{B_{1}} v_{\delta} \nabla \eta \cdot \nabla v_{0} d x+\int_{B_{1}} \eta\left|\nabla v_{\delta}\right|^{2} d x=0
$$

and letting $\delta \rightarrow 0$, we get

$$
\int_{B_{1}} v_{0} \nabla \eta \cdot \nabla v_{0} d x+\int_{B_{1}} \eta\left|\nabla v_{0}\right|^{2} d x=0
$$

Using (4.2), we obtain

$$
\limsup _{\epsilon_{i} \rightarrow 0} \int_{B_{1}} \eta\left|\nabla v_{\epsilon_{i}}\right|^{2} d x \leq \int_{B_{1}} \eta\left|\nabla v_{0}\right|^{2} d x .
$$

Combining (4.1) and (4.3), we obtain

$$
\lim _{\epsilon_{i} \rightarrow 0} \int_{B_{1}} \eta\left|\nabla v_{\epsilon_{i}}\right|^{2} d x=\int_{B_{1}} \eta\left|\nabla v_{0}\right|^{2} d x,
$$

which implies that

$$
\eta^{1 / 2} \nabla v_{\epsilon_{i}} \rightarrow \eta^{1 / 2} \nabla v_{0} \text { in } L^{2}\left(B_{1}\right)
$$

For $\eta$ such that $\eta=1$ in $D \subset \subset \Omega$, it follows that

$$
\nabla v_{\epsilon_{i}} \rightarrow \nabla v_{0} \text { in } L^{2}(D)
$$

Therefore $v_{\epsilon} \rightarrow v_{0}$ strongly in $W^{1,2}(D)$.

By a diagonal sequence argument, assertion 2) of the proposition follows.

3) Since $v_{0}$ is continuous, the sets $\left\{v_{0}>0\right\}$ and $\left\{v_{0}<0\right\}$ are open. Let $x^{0} \in\left\{v_{0}>0\right\}$. From the fact that $v_{\epsilon_{i}} \rightarrow v_{0}$ uniformly on $B_{1}$, there exist $r>0$ and $N \in \mathbb{N}$ such that $v_{\epsilon_{i}}(x) \geq v_{0}\left(x^{0}\right) / 2>0$ for $x \in B_{r}\left(x^{0}\right)$, $i \geq N$. Thus $v_{\epsilon_{i}}$ is harmonic in $B_{r}\left(x^{0}\right)$ for $i \geq N$ and the same fact holds for $v_{0}$. In the same way we prove that $v_{0}$ is superharmonic in $\left\{v_{0} \leq 0\right\}^{0}$. On the other hand, $v_{\epsilon_{i}}$ is subharmonic in $B_{1}$, so that $v_{0}$ is also subharmonic. Therefore $v_{0}$ is harmonic in $\left\{v_{0} \leq 0\right\}^{0}$ and 3$)$ follows. 
4) Multiplying equation $\Delta v_{\epsilon_{i}}=\beta_{\epsilon_{i}}\left(v_{\epsilon_{i}}\right)$ by $\phi \in C_{0}^{\infty}\left(B_{1}\right)$ and integrating by parts, we get

$$
\int_{B_{1}} \nabla v_{\epsilon_{i}} \cdot \nabla \phi d x+\int_{B_{1}} \beta_{\epsilon_{i}}\left(v_{\epsilon_{i}}\right) \phi d x=0 .
$$

We know that $v_{\epsilon_{i}} \rightarrow v_{0}$ in $W_{\text {loc }}^{1,2}\left(B_{1}\right)$, hence

$$
\int_{B_{1}} \nabla v_{\epsilon_{i}} \cdot \nabla \phi d x \rightarrow \int_{B_{1}} \nabla v_{0} \cdot \nabla \phi d x .
$$

On the other hand,

$$
\int_{B_{1}} \beta_{\epsilon_{i}}\left(v_{\epsilon_{i}}\right) \phi d x \leq C
$$

This $L^{1}$-bound implies that there exists a locally finite non-negative measure $\mu$ such that for a subsequence which we still call $\left\{v_{\epsilon_{i}}\right\}, \beta_{\epsilon_{i}}\left(v_{\epsilon_{i}}\right) \rightarrow$ $\mu$ as measures in $B_{1}$. Passing to the limit in (4.5), we get

$$
\int_{B_{1}} \nabla v_{0} \cdot \nabla \phi d x+\int_{B_{1}} \phi d \mu=0
$$

which implies

$$
\Delta v_{0}=\mu \quad \text { in } B_{1} .
$$

Since we know that $v_{0}$ is harmonic in $B_{1} \backslash \partial\left\{v_{0}>0\right\}$, we conclude that supp $\mu \subset \partial\left\{v_{0}>0\right\}$.

5) follows from the fact that $v_{\epsilon_{i}}$ has been defined via even reflection. This property is preserved by the uniform limit $v_{0}$.

Let $\mathcal{B}_{\epsilon}(z)=\int_{0}^{z} \beta_{\epsilon}(s) d s$ and $\chi_{\epsilon}(x)=2 \mathcal{B}_{\epsilon}\left(v_{\epsilon}(x)\right)$. It follows that $0 \leq \chi_{\epsilon}(x) \leq 1$, and the uniformly Lipschitz estimate for $v_{\epsilon}$ implies the relative compactness of $\chi_{\epsilon}$ in $L_{\text {loc }}^{1}\left(B_{1}\right)$ :

Proposition 4.4. $\left\{\chi_{\epsilon}\right\}$ is precompact in $L^{1}(D)$ for each $D \subset \subset B_{1}$.

Proof. By Proposition 4.1, there exists an $L>0$ such that $\left\|\nabla v_{\epsilon}\right\|_{L^{\infty}(D)} \leq$ $L$, hence

$$
\int_{D}\left|\nabla \chi_{\epsilon}\right| d x=2 \int_{D}\left|\beta_{\epsilon}\left(v_{\epsilon}\right) \nabla v_{\epsilon}\right| d x \leq 2 L \int_{D} \beta_{\epsilon}\left(v_{\epsilon}\right) d x \leq C .
$$

Therefore $\left\{\chi_{\epsilon}\right\}$ is bounded in $W^{1,1}(D)$. By the Sobolev embedding theorem $\left\{\chi_{\epsilon}\right\}$ is precompact in $L^{1}(D)$. 
Thus we may assume that $\chi_{\epsilon_{i}}(x) \rightarrow \chi_{0}(x)$ locally in $L^{1}$. Similar to [12, Lemma 4.1], we have

Proposition 4.5. $\chi_{0}(x) \in\{0,1\}$ for a.e. $x \in B_{1}$.

Proof. For $\delta \in(0,1 / 4), \mathcal{B}(s)=2 \int_{0}^{s} \beta(t) d t$ and $A_{\delta}=\left\{x \in D \subset \subset B_{1}\right.$ : $\left.\chi_{0} \in[2 \delta, 1-2 \delta]\right\}$ we have

$$
\begin{aligned}
\mathcal{L}^{2}\left(A_{\delta}\right) & \leq \mathcal{L}^{2}\left(A_{\delta} \cap\left\{\chi_{\epsilon_{m}} \notin[\delta, 1-\delta]\right\}\right)+\mathcal{L}^{2}\left(A_{\delta} \cap\left\{\mathcal{B}\left(\frac{v_{\epsilon_{m}}}{\epsilon_{m}}\right) \in[\delta, 1-\delta]\right\}\right) \\
& \leq \mathcal{L}^{2}\left(D \cap\left\{\left|\chi_{\epsilon_{m}}-\chi_{0}\right|>\delta\right\}\right)+\mathcal{L}^{2}\left(D \cap\left\{\frac{v_{\epsilon_{m}}}{\epsilon_{m}} \in\left[\mathcal{B}^{-1}(\delta), \mathcal{B}^{-1}(1-\delta)\right]\right\}\right) \\
& \leq \mathcal{L}^{2}\left(D \cap\left\{\left|\chi_{\epsilon_{m}}-\chi_{0}\right|>\delta\right\}\right)+C_{1}(\delta, \beta) \epsilon_{m} \int_{D} \beta_{\epsilon_{m}}\left(v_{\epsilon_{m}}\right) d x \\
& \leq \mathcal{L}^{2}\left(D \cap\left\{\left|\chi_{\epsilon_{m}}-\chi_{0}\right|>\delta\right\}\right)+C_{2}(\delta, \beta) \epsilon_{m} \rightarrow 0
\end{aligned}
$$

as $m \rightarrow \infty$.

\section{Monotonicity Formula}

In this section we state a monotonicity formula proved in [13], which is a key tool in constructing a degenerate singularity.

Proposition 5.1. Let $x^{0} \in B_{1}$, and

$$
\Phi_{\epsilon}(r)=r^{-2} \int_{B_{r}\left(x^{0}\right)}\left|\nabla v_{\epsilon}\right|^{2}+r^{-2} \int_{B_{r}\left(x^{0}\right)} \chi_{\epsilon}-r^{-3} \int_{\partial B_{r}\left(x^{0}\right)} v_{\epsilon}^{2} d \mathcal{H}^{1} .
$$

Then $\Phi_{\epsilon}$ satisfies the monotonicity formula

$\Phi_{\epsilon}(\sigma)-\Phi_{\epsilon}(\rho) \geq \int_{\rho}^{\sigma} r^{-2} \int_{\partial B_{r}\left(x^{0}\right)} 2\left(\nabla v_{\epsilon} \cdot \nu-\frac{v_{\epsilon}(x)}{r}\right)^{2} d \mathcal{H}^{1} d r$ for $0<\rho<\sigma$.

Letting $\epsilon \rightarrow 0$,

$$
\Phi(r)=r^{-2} \int_{B_{r}\left(x^{0}\right)}\left|\nabla v_{0}\right|^{2}+r^{-2} \int_{B_{r}\left(x^{0}\right)} \chi_{0}-r^{-3} \int_{\partial B_{r}\left(x^{0}\right)} v_{0}^{2} d \mathcal{H}^{1}
$$

satisfies the monotonicity formula

$$
\Phi(\sigma)-\Phi(\rho) \geq \int_{\rho}^{\sigma} r^{-2} \int_{\partial B_{r}\left(x^{0}\right)} 2\left(\nabla v_{0} \cdot \nu-\frac{v_{0}(x)}{r}\right)^{2} d \mathcal{H}^{1} d r \text { for } 0<\rho<\sigma .
$$




\section{Existence of a Degenerate Free Boundary Point}

In this section we are going to prove the existence of a degenerate singular free boundary point for $v_{0}$. We start with some lemmas.

Lemma 6.1. Let $w$ be a solution of

$$
\Delta w=\beta_{\epsilon}(w) \text { in } \Omega \subset \mathbb{R}^{n} \text { and let } \chi_{\epsilon}(x)=2 \mathcal{B}_{\epsilon}(w(x)) \text {. }
$$

Then

$$
\int_{\Omega}|\nabla w|^{2} \operatorname{div} \phi-2 \nabla w D \phi \nabla w+\chi_{\epsilon} \operatorname{div} \phi d x=0
$$

for $\phi \in C_{0}^{1}\left(\Omega ; \mathbb{R}^{n}\right)$.

Proof. Integrating by parts, we get

$$
\begin{aligned}
& \int_{\Omega}|\nabla w|^{2} \operatorname{div} \phi-2 \nabla w D \phi \nabla w+\chi_{\epsilon} \operatorname{div} \phi d x \\
& =\int_{\Omega} \sum_{i=1}^{n}\left(\frac{\partial w}{\partial x_{i}}\right)^{2} \sum_{j=1}^{n} \frac{\partial \phi_{j}}{\partial x_{j}}-2 \sum_{i, j=1}^{n} \frac{\partial w}{\partial x_{i}} \frac{\partial \phi_{j}}{\partial x_{i}} \frac{\partial w}{\partial x_{j}}+\chi_{\epsilon} \operatorname{div} \phi d x \\
& =-2 \int_{\Omega} \sum_{i, j=1}^{n} \phi_{j} \frac{\partial w}{\partial x_{i}} \frac{\partial^{2} w}{\partial x_{i} \partial x_{j}}-\sum_{i, j=1}^{n} \phi_{j} \frac{\partial}{\partial x_{i}}\left(\frac{\partial w}{\partial x_{i}} \frac{\partial w}{\partial x_{j}}\right)+\beta_{\epsilon}(w) \nabla w \cdot \phi d x \\
& =2 \int_{\Omega} \sum_{i, j=1}^{n} \phi_{j} \frac{\partial w}{\partial x_{j}} \frac{\partial^{2} w}{\partial x_{i}^{2}}-\beta_{\epsilon}(w) \nabla w \cdot \phi d x \\
& =2 \int_{\Omega} \nabla w \cdot \phi\left(\Delta w-\beta_{\epsilon}(w)\right) d x \\
& =0 .
\end{aligned}
$$

Lemma 6.2. If $v_{0} \equiv 0$ in $B_{r}$ for some $r>0$, then $\chi_{0} \equiv 1$ in $B_{r}$.

Proof. By Lemma 6.1 we have

$$
\int_{B_{1}}\left|\nabla v_{\epsilon_{i}}\right|^{2} \operatorname{div} \phi-2 \nabla v_{\epsilon_{i}} D \phi \nabla v_{\epsilon_{i}}+\chi_{\epsilon_{i}} \operatorname{div} \phi d x=0
$$




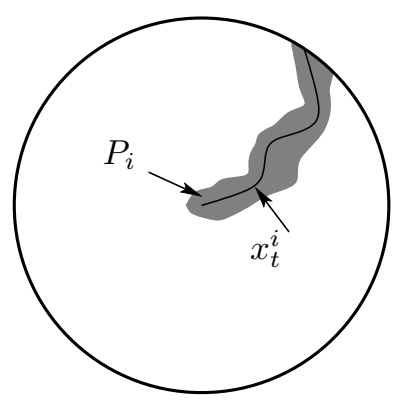

Figure 4 . The set $P_{i}$

for $\phi \in C_{0}^{\infty}\left(B_{r} ; \mathbb{R}^{2}\right)$. Letting $\epsilon_{i} \rightarrow 0$, we obtain from the strong convergence of $v_{\epsilon_{i}}$ that

$$
\begin{aligned}
0 & =\int_{B_{1}}\left|\nabla v_{0}\right|^{2} \operatorname{div} \phi-2 \nabla v_{0} D \phi \nabla v_{0}+\chi_{0} \operatorname{div} \phi d x \\
& =\int_{B_{r}} \chi_{0} \operatorname{div} \phi d x,
\end{aligned}
$$

hence $\chi_{0} \equiv$ const in $B_{r}$. We know that $\chi_{0}(x) \in\{0,1\}$ for a.e. $x \in B_{1}$, therefore $\chi_{0}(x) \equiv 0$ or $\chi_{0}(x) \equiv 1$ in $B_{r}$.

For $0<c \leq 1 / 4$, let $P_{i}=\left\{x \in B_{r}: c \epsilon_{i} \leq v_{\epsilon_{i}}(x) \leq(1-c) \epsilon_{i}\right\}$ and $Q_{i}=\left\{x \in B_{r}: v_{\epsilon_{i}}(x)>c \epsilon_{i}\right\}$. Supposing towards a contradiction that $\chi_{0} \equiv 0$ in $B_{r}$, and recalling that $\chi_{\epsilon_{i}} \rightarrow \chi_{0}$ in $L^{1}\left(B_{r}\right)$ we have

$$
\mathcal{L}^{2}\left(Q_{i}\right) \leq \mathcal{L}^{2}\left(\left\{x: \chi_{\epsilon_{i}}>2 \mathcal{B}(c)\right\}\right) \rightarrow 0
$$

as $i \rightarrow \infty$.

Let $\delta \in(0, r / 4), I_{\epsilon_{i}}=\left\{t: \delta \leq t \leq r, \min _{x \in \partial B_{t}} v_{\epsilon_{i}}(x) \geq \epsilon_{i} / 2\right\}$ and $I_{\epsilon_{i}}^{c}=[\delta, r] \backslash I_{\epsilon_{i}}$. Then we have $\mathcal{L}^{1}\left(I_{\epsilon_{i}}\right) \leq \mathcal{L}^{2}\left(Q_{i}\right) / \delta \rightarrow 0$ as $i \rightarrow \infty$, hence there exists an $\epsilon_{0}>0$ such that $\mathcal{L}^{1}\left(I_{\epsilon_{i}}^{c}\right)>r / 2$ for $\epsilon_{i}<\epsilon_{0}$. As $v_{\epsilon_{i}}$ is subharmonic in $B_{1}, \epsilon_{i}=v_{\epsilon_{i}}(0) \leq \max _{\partial B_{t}} v_{\epsilon_{i}}$ for every $t \in[0, r)$. It follows that for each $t \in I_{\epsilon_{i}}^{c}$, there exists a point $x_{t}^{i} \in \partial B_{t}$ such that $v_{\epsilon_{i}}\left(x_{t}^{i}\right)=\epsilon_{i} / 2$ (cf. Figure 4). By the uniformly Lipschitz continuity of $v_{\epsilon_{i}}$ there exists a $d>0$ independent of $\epsilon$, such that $B_{d \epsilon_{i}}\left(x_{t}^{i}\right) \subset P_{i}$. Therefore

$$
\mathcal{L}^{2}\left(P_{i}\right) \geq \mathcal{L}^{2}\left(\bigcup_{x_{t}^{i} \in I_{\epsilon_{i}}^{c}} B_{d \epsilon_{i}}\left(x_{t}^{i}\right)\right) \geq r d \epsilon_{i} / 4
$$


On the other hand, since $\Delta v_{\epsilon_{i}} \rightarrow 0$ in $B_{r}$,

$$
\mathcal{L}^{2}\left(P_{i}\right) / \epsilon_{i} \leq C \int_{B_{r}} \beta_{\epsilon_{i}}\left(v_{\epsilon_{i}}\right) \rightarrow 0 .
$$

Thus we get a contradiction and therefore $\chi_{0} \equiv 1$ in $B_{r}$.

Lemma 6.3. Let $v_{0}\left(x^{0}\right)=0$ for some $x^{0} \in B_{1}, 0<r_{k} \rightarrow 0$ as $k \rightarrow \infty$ and $v_{0 k}(x)=\frac{v_{0}\left(x^{0}+r_{k} x\right)}{r_{k}}$. Then there exists a blow-up limit $v_{00}$ such that for a subsequence the following holds:

1) $v_{0 k} \rightarrow v_{00}$ in $C_{\text {loc }}^{0, \alpha}\left(\mathbb{R}^{2}\right)$ for every $0<\alpha<1, \nabla v_{0 k} \rightarrow \nabla v_{00}$ weakly* in $L_{\mathrm{loc}}^{\infty}\left(\mathbb{R}^{2}\right)$,

2) $v_{0 k} \rightarrow v_{00}$ in $W_{\mathrm{loc}}^{1,2}$,

3) $v_{00}$ is homogeneous of degree 1 .

Proof. 1) Since $\nabla v_{0 k}(x)=\nabla v_{0}\left(x_{0}+r_{k} x\right),\left\{v_{0 k}\right\}$ is locally uniformly Lipschitz continuous in $\mathbb{R}^{2}$ and 1 ) follows.

2) Let $D \subset \subset \mathbb{R}^{2}$. Then there exists a $C<\infty$ such that $\left|\nabla v_{0 k}(x)\right|<$ $C$ in $D$, hence $v_{0 k}$ is bounded $W^{1,2}(D)$. It follows that there is a subsequence, which we still call $v_{0 k}$, such that $v_{0 k} \rightarrow v_{00}$ weakly in $W^{1,2}(D)$ and

$$
\left\|v_{00}\right\|_{W^{1,2}(D)} \leq \liminf _{k \rightarrow \infty}\left\|v_{0 k}\right\|_{W^{1,2}(D)} .
$$

Since $\Delta v_{0}=\mu$ and supp $\mu \subset \partial\left\{v_{0}>0\right\}$, we get

$$
v_{0 k}(x) \Delta v_{0 k}(x)=0 .
$$

Let $\eta \in C_{0}^{\infty}\left(\mathbb{R}^{2}\right)$. Then $\int_{\mathbb{R}^{2}} \eta\left|\nabla v_{0 k}\right|^{2}=-\int_{\mathbb{R}^{2}} v_{0 k} \nabla \eta \cdot \nabla v_{0 k}$ and

$$
\limsup _{k \rightarrow 0} \int_{\mathbb{R}^{2}} \eta\left|\nabla v_{0 k}\right|^{2} \leq-\int_{\mathbb{R}^{2}} v_{00} \nabla \eta \cdot \nabla v_{00} .
$$

For each $\delta>0$, we define

$$
v_{\delta}(x)= \begin{cases}v_{00}(x)-\delta, & \text { if } v_{00}(x)>\delta, \\ v_{00}(x)+\delta, & \text { if } v_{00}(x)<-\delta, \\ 0 & \text { otherwise. }\end{cases}
$$

Multiplying the equation for $v_{0 k}$ by $\eta v_{\delta}$, we get

$$
\eta(x) v_{\delta}(x) \Delta v_{0 k}(x)=0,
$$


if $k$ is large enough. Hence

$$
\int_{\mathbb{R}^{2}} \eta \nabla v_{\delta} \cdot \nabla v_{0 k}=-\int_{\mathbb{R}^{2}} v_{\delta} \nabla \eta \cdot \nabla v_{0 k}
$$

Letting $k \rightarrow \infty$, it follows that

$$
\int_{\mathbb{R}^{2}} \eta \nabla v_{\delta} \cdot \nabla v_{00}=-\int_{\mathbb{R}^{2}} v_{\delta} \nabla \eta \cdot \nabla v_{00},
$$

and letting $\delta \rightarrow 0$, we get

$$
\int_{\mathbb{R}^{2}} \eta\left|\nabla v_{00}\right|^{2}=-\int_{\mathbb{R}^{2}} v_{00} \nabla \eta \cdot \nabla v_{00}
$$

Hence

$$
\limsup _{k \rightarrow 0} \int_{\mathbb{R}^{2}} \eta\left|\nabla v_{0 k}\right|^{2} \leq \int_{\mathbb{R}^{2}} \eta\left|\nabla v_{00}\right|^{2}
$$

for every $\eta \in C_{0}^{\infty}\left(\mathbb{R}^{2}\right)$. It follows that $v_{0 k} \rightarrow v_{00}$ in $W^{1,2}(D)$.

3) For $0<R<S<\infty$,

$$
\Phi\left(R r_{k}\right)=R^{-2} \int_{B_{R}}\left|\nabla v_{0 k}\right|^{2}+\chi_{0}\left(r_{k} x\right) d x-R^{-3} \int_{\partial B_{R}} v_{0 k}^{2} d \mathcal{H}^{1} .
$$

Since $v_{0}\left(x^{0}\right)=0$ and $v_{0}$ is Lipschitz continuous, $\Phi(r)$ is bounded. Consequently we obtain from the monotonicity formula Proposition 5.1 that

$$
\begin{aligned}
0 \leftarrow \Phi\left(S r_{k}\right)-\Phi\left(R r_{k}\right) & =\int_{R}^{S} 2 r^{-2} \int_{\partial B_{r}}\left(\nabla v_{0 k} \cdot \nu-\frac{v_{0 k}}{r}\right)^{2} d \mathcal{H}^{1} d r \\
& =\int_{B_{S} \backslash B_{R}} 2|x|^{-4}\left(\nabla v_{0 k}(x) \cdot x-v_{0 k}(x)\right)^{2} d x
\end{aligned}
$$

Letting $k \rightarrow \infty$, we obtain that $\nabla v_{00}(x) \cdot x=v_{00}(x)$ a.e. in $\mathbb{R}^{2}$, hence $v_{00}$ is homogeneous of degree 1 .

Now we are ready to prove Theorem A of the Introduction.

Theorem 6.4. There is a free boundary point $x^{0} \in \partial\left\{v_{0}>0\right\}$ such that $v_{0}$ is degenerate at $x^{0}$. More precisely,

$$
\lim _{r \rightarrow 0} \frac{v_{0}\left(x^{0}+r x\right)}{r}=0
$$

for every $x \in \mathbb{R}^{2}$. 


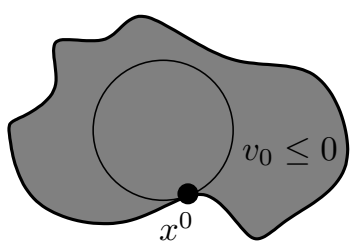

Figure 5. Touching the Free Boundary

Proof. Case 1: $0 \notin \partial\left\{v_{0}>0\right\}$.

There exists an $r>0$ such that $v_{0}(x) \leq 0$ in $B_{r}$. Since $v_{0}(0)=0$, the subharmonicity of $v_{0}$ implies that $v_{0} \equiv 0$ in $B_{r}$. Suppose that $r$ is the largest number such that $v_{0} \equiv 0$ in $B_{r}$. Noticing that $r<1$ as the boundary values of $v_{0}$ are not constant. It follows that there is a point $x^{0} \in \partial B_{r}$ such that $x^{0} \in \partial\left\{v_{0}>0\right\}$ (cf. Figure 5 ).

By a translation and a rotation we may assume that $x^{0}=0$ and $v_{0} \equiv 0$ in $B_{r}(-r, 0)$. Let $v_{00}$ be a blow-up limit of $v_{0}$ at $x^{0}$, i.e. the limit of $\frac{v_{0}\left(x^{0}+r_{k} x\right)}{r_{k}}$ as $k \rightarrow \infty$. We are going to show that $v_{00} \equiv 0$.

Suppose towards a contradiction that $v_{00} \not \equiv 0$ and let $S=\left\{v_{00}>0\right\}$. Then $v_{00}$ is harmonic in $S$. Moreover, we know from Lemma 6.3 that $v_{00}$ is homogeneous of degree 1 , so that, solving the resulting ODE for $v_{00}, v_{00}=c x \cdot \nu$ in $S$ for some $c \in \mathbb{R}$ and $\nu \in \partial B_{1}$. Noticing that $v_{00} \equiv 0$ in the left half plane, we conclude that $v_{00}=c \max (0, x \cdot \nu)$, where $c>0$ and $\nu=(1,0)$.

Let $\chi_{r}(x)=\chi_{0}(r x)$ and let $\chi_{00}$ be the limit of $\chi_{r_{k}}$ of the above sequence $k \rightarrow \infty$. By Lemma 6.2, $\chi_{0} \equiv 1$ in $B_{r}(-r, 0)$, hence $\chi_{00} \equiv 1$ in the left half plane. Moreover we know that $\chi_{0}(x)=1$ if $v_{0}(x)>0$, hence $\chi_{00} \equiv 1$ in the right half plane. Thus $\chi_{00}(x) \equiv 1$ in $\mathbb{R}^{2}$.

From

$$
0=\int_{\mathbb{R}^{2}}\left|\nabla v_{00}\right|^{2} \operatorname{div} \phi-2 \nabla v_{00} D \phi \nabla v_{00}+\chi_{00} \operatorname{div} \phi d x
$$

for every $\phi \in C_{0}^{\infty}\left(\mathbb{R}^{2} ; \mathbb{R}^{2}\right)$, we infer that

$$
\int_{\mathbb{R}^{2}}\left|\nabla v_{00}\right|^{2} \operatorname{div} \phi-2 \nabla v_{00} D \phi \nabla v_{00}=0
$$


for every $\phi \in C_{0}^{\infty}\left(\mathbb{R}^{2} ; \mathbb{R}^{2}\right)$. This however contradicts $v_{00}=c \max (0, x$. $\nu)$. We conclude that the blow-up limit $v_{00}$ must be the constant function 0 in $\mathbb{R}^{2}$.

Case 2: $0 \in \partial\left\{v_{0}>0\right\}$.

Suppose towards a contradiction that a blow-up limit

$$
v_{00}=\lim _{j \rightarrow \infty} \frac{v_{0}\left(s_{j}\right)}{s_{j}} \not \equiv 0 .
$$

Similar to case $1, v_{00}$ must be of the form $c x \cdot \nu$ in some half plane; here $\nu$ is again a unit vector. On the other hand Proposition 4.35) - which incidentally is preserved under the blow-up limit - implies that there are at least two vectors $e^{1} \neq e^{2} \in \partial B_{1}$ and two half lines $\left\{\alpha e^{1}: \alpha>0\right\}$ and $\left\{\alpha e^{2}: \alpha>0\right\}$, such that $e^{1} \cdot \nu>0, \nabla v_{00}=\nabla v_{00} \cdot e^{1} e^{1}, e^{2} \cdot \nu>0$ and $\nabla v_{00}=\nabla v_{00} \cdot e^{2} e^{2}$ which is not possible for $v_{00}(x)=c x \cdot \nu$ unless $c=0$.

Remark 6.5. Using methods in the forthcoming paper [11, it is actually possible to show that Case 1 in the proof of Theorem 6.4 cannot occur. More precisely, a degenerate point $x^{0}$ at which the set $\left\{v_{0}=0\right\}$ contains a disk touching $x^{0}$ is not possible.

Consequently the origin must in our example be a degenerate point. The fact that we can introduce many symmetry lines suggests that we can construct degenerate points with growth $\leq|x|^{m}$ in $B_{1 / 2}$ for any integer $m$.

\section{REFERENCES}

[1] H. W. Alt and L. A. Caffarelli. Existence and regularity for a minimum problem with free boundary. J. Reine Angew. Math., 325:105-144, 1981.

[2] J. Andersson and G. S. Weiss. Cross-shaped and degenerate singularities in an unstable elliptic free boundary problem. J. Differential Equations, 228(2):633640, 2006.

[3] H. Berestycki, L. A. Caffarelli, and L. Nirenberg. Uniform estimates for regularization of free boundary problems. In Analysis and partial differential equations, volume 122 of Lecture Notes in Pure and Appl. Math., pages 567-619. Dekker, New York, 1990.

[4] J. D. Buckmaster and G. S. S. Ludford. Theory of laminar flames. Cambridge Monographs on Mechanics and Applied Mathematics. Cambridge University 
Press, Cambridge, 1982. Electronic \& Electrical Engineering Research Studies: Pattern Recognition \& Image Processing Series, 2.

[5] Luis A. Caffarelli. Uniform Lipschitz regularity of a singular perturbation problem. Differential Integral Equations, 8(7):1585-1590, 1995.

[6] D. Danielli, A. Petrosyan, and H. Shahgholian. A singular perturbation problem for the $p$-Laplace operator. Indiana Univ. Math. J., 52(2):457-476, 2003.

[7] J. Frehse. Capacity methods in the theory of partial differential equations. Jahresber. Deutsch. Math.-Verein., 84(1):1-44, 1982.

[8] David Gilbarg and Neil S. Trudinger. Elliptic partial differential equations of second order, volume 224 of Grundlehren der Mathematischen Wissenschaften [Fundamental Principles of Mathematical Sciences]. Springer-Verlag, Berlin, second edition, 1983.

[9] Alex Gurevich. Boundary regularity for free boundary problems. Comm. Pure Appl. Math., 52(3):363-403, 1999.

[10] Claudia Lederman and Noemi Wolanski. Viscosity solutions and regularity of the free boundary for the limit of an elliptic two phase singular perturbation problem. Ann. Scuola Norm. Sup. Pisa Cl. Sci. (4), 27(2):253-288 (1999), 1998.

[11] E. Varvaruca and G. S. Weiss. A geometric proof of a generalized Stokes conjecture. In preparation.

[12] G. S. Weiss. A singular limit arising in combustion theory: fine properties of the free boundary. Calc. Var. Partial Differential Equations, 17(3):311-340, 2003.

[13] Georg S. Weiss. Partial regularity for weak solutions of an elliptic free boundary problem. Comm. Partial Differential Equations, 23(3-4):439-455, 1998.

Graduate School of Mathematical Sciences, University of Tokyo, 3-8-1 Komaba, Meguro-ku, Tokyo-to, 153-8914 Japan

E-mail address: gw@ms.u-tokyo.ac.jp

Graduate School of Mathematical Sciences, University of Tokyo, 3-8-1 Komaba, Meguro-Ku, Tokyo-to, 153-8914 Japan

E-mail address: zhang@ms.u-tokyo.ac.jp 